\title{
vCloud Analytics for Capacity Planning
}

\author{
A.V.K.Shanthi, \\ Faculty of Computing, Sathyabama University, Chennai, India, \\ avks15@gmail.com.
}

Abstract-

Cloud Computing is ubiquitous computing, in which we can be able to access the computing resources, shared storage, network by on demand.Cloud computing has a part on virtualization technology, in which it provides the end user with server consolidation. The advantage of using Cloud is, it requires less hardware requirement and gives us greater flexibility. When the services are executing by on demand using the Cloud resources, here comes the resource inadequate for the user, and for this solution the providers make use of the Cloud Capacity planning.vCloud Capacity planner is an automated performance analytics for provisioning objects in dynamic Virtual and Cloud environments. In vCloud Capacity planner we had analysed with VMware vSphere (provides virtual infrastructure) environment with real-time data.

\section{INTRODUCTION}

Capacity planning and management is an vital source for an organization to provide a consistent resources like compute, storage, network and etc., available for the enterprise, all time by saving the cost of organization. This paper will experimentally explains how the cloud and virtual infrastructure resources have been functionally take cares by the cloud capacity planner in VMware cloud technology. In general the cloud administrator, who is responsible for the organization's capacity planning has to quote for future plan to execute the changes in capacity and purchase of hardware and virtual resources needed for the particular datacenter usage in proper time, before the consumption of resource arises. Herecomes the need for capacity planner for cloud models to provide the efficient planning for resource utilization and cost effective. Capacity Planner in vCloud alerts us, if we are running the low infrastructure virtualization resources like memory,CPU, storage capacity and storage throughput levels in virtual machines, datastore, hosts, multiple datacenters, clusters etc..,vCloud Capacity planner has been classified into [1]Analyzing, [2]Optimizing, [3]Forecasting.Using this property the vCloud capacity planner provides graphical representation for the events happening in the virtual infrastructure as risk, time. Capacity, stress, waste, efficiency, and density level.

\section{RELATED WORK}

The increased utility of the resource and extensibility of modern computing server or system technology made the far difficult in measuring the utilization of resources in virtual and cloud environment. The Cost of SLA and resource functionality discussed in [4],[11] y.Zinng derived the formulation for analyzing consumption of resource utilization and wastage of resource during new server provisioning. Cost Optimization and efficient usage of the resource were discussed in [10],[13].Two Phase service algorithm were also proposed in the above mentioned paper, for the resource monitoring and efficient usage of the provisioning resources. The contemption of resource in the particular dataceter leads to delay in provisioning Virtual Machine, thus handling such incoming demands from the cloud user supports to the capacity planning request fulfillment has discussed in $[8],[7],[12]$.

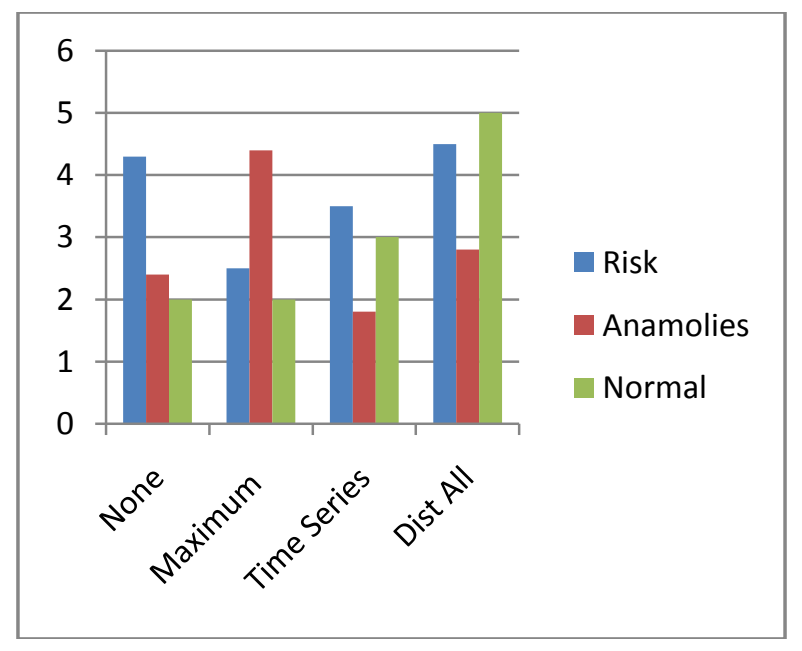

Fig 1. Using Normal Distribution

The Prediction approach for provisioning and deprovisioning resource to the virtual machine in the 
[12],[17] still delimits the risk facts of the virtual machine. The instant provisioning of virtual machine in the [12] relates the SLA for over provisioning and under provisioning of the virtual and cloud providers leads to pay penalty forviolating the SLA's. Admission control explained in the [15],[8] thus controls the provisioning of resources according to the acceptance or rejection of on demand requests from user. Data Filtering and aggregation uses the data preprocessing algorithm which predicts evaluates the irregularity of virtual machine fuctions due to the over-provisioning[6],[10].

\section{PROPOSED WORK}

All related approached work gives the traditional way for enterprise capacity planner, rather than giving modernized planning [12]. The business need have to move at the speed by rapid provisioning of infrastructure service and must scale up the IT Operations by providing the on demand user to access scaled capacity on demand. vCloud capacity planner in the proposed model delivers analyzed, planned and forecasted reports as well as the operation to do for extending the efficiency of particular environment. And the most important feature of vCloud capacity planner has alerts, different types of badges, stress, workload anomalies, efficiency for different resource consumptions of the datacenter objects. The algorithm described in [12] uses normal distribution to display the statistics of data segment in virtual and cloud infrastructure. Thus Dynamic Threshold algorithm provide the root cause for causing abnormalities of data splitting. Average shifting algorithm ASH used in[16],model describes the traditional analysis for data segmentation in the environment.

\section{Dynamic Threshold}

vCloud Capacity planner defines the dynamic threshold for every data collection based upon the statistics defined by the historical representation. Dynamic threshold tracks the normal and abnormal values and sets a time interval, which uses a analytic algorithm. Dynamic threshold algorithm used to detect linear behaviourpattern, different metrics that have discrete set of values not a range of values, cyclical behavior patterns, multi-model. Dynamic threshold algorithm does not work with time series or frequently measured values as the traditional, but works with sparse data. The Graph below shows the approach for detecting cyclical behavior patterns, metrics graphs

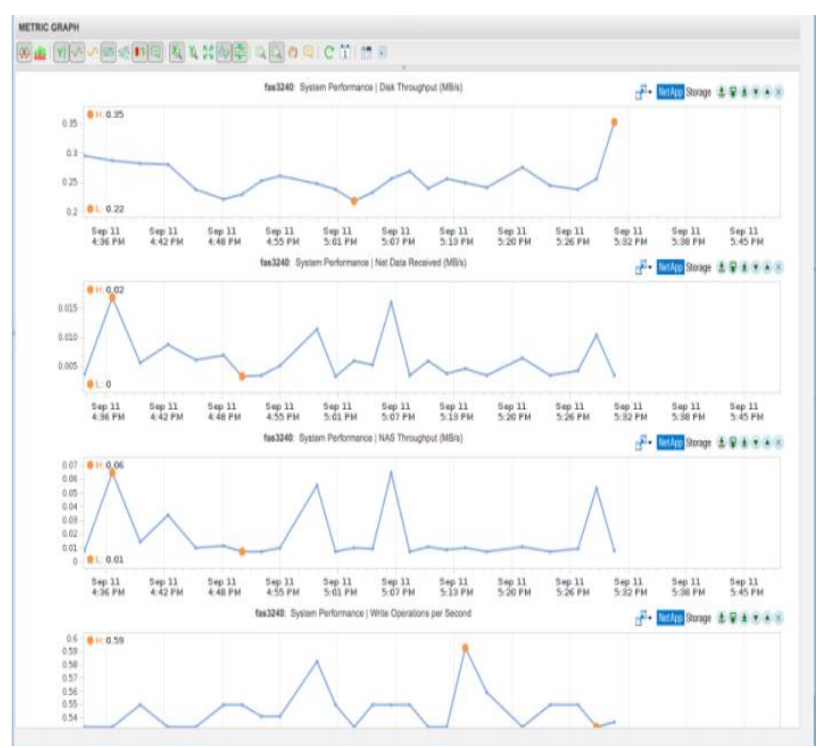

Fig 2. Using Dynamic Threshold

\section{System Architecture}

The System Architecture of vCloud capacity planner is composed of Cluster node, in which it contains the vAPP (Collection of virtual machine bundled together), Data store and network in which it points to give the stastical report of VM running on particular datacenter.

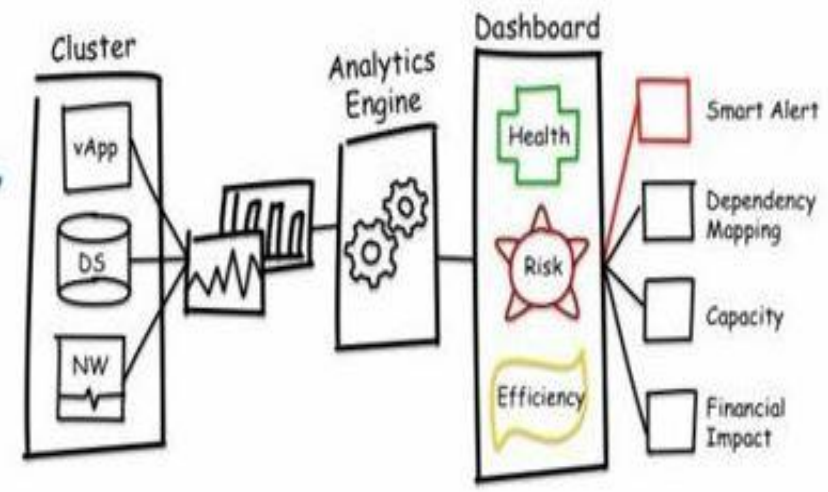

Fig.3 System Architecture

The architecture cluster consist of UI VM and Analytic VM. Using the UI VM we can access the results of analytics in the form of badges, and health status and Analytic VM is used to collect data from particular virtualized or cloud environment, and store the raw data's in scalable File system database. The analytic VM has the capacity and performance analytics, capacity collector, and also uses the PostgreSQL database. The main functionality of vCloud capacity planner is explained briefly below.

Attributes and Metrics - vCloud Capacity planner collects the data of CPU, memory, storage, and hard disk etc..., The type of data that collects from each object is 
called attribute. And each piaece of data that the vCloud manager collects is called metrics.

Hard Threshold - Unlike a Dynamic threshold we could not set a floating value for metric, hard threshold uses a fixed value to define the normal behavior for an objects.

Key performance indicators - vCloud Capacity planner defines the attribute which are performing as a critical object collectively called as key Performance Indicators (KPI)

Alerts and Faults -vCloud capacity Manager generates the alarm when data analysis deviates from the normal metric values. Capacity management can be divided into Analyzing, which focuses immediate or long term capacity issues, Optimizing which focuses the virtual machine to consume more resource utilization and minimize the unwanted wastage resources, Forecasting is traditional capacity planner which predicts forecoming capacity issues in datacenter.

\section{EXPERIMENTAL RESULT}

vCloud Capacity planner monitors the resource utilization using the analytic engine described in system architecture and store in file system database in which the real-time experimental dashboard of the dynamic threshold algorithm will looks as follows, in fig 5.shows the dashboard in which it contains the reports, planning, operations, alerts, analysis, environment and alerts.

In the object panel it shows the datastores, hosts, virtual machine, vApps, and cluster health status in the given VMware vSphere environment. The Dashboard triggers the alarm notification for the user if the resource gets consumed to least level using badges, it also has the badges for health, risk, and efficiencyand also using the dynamic threshold algorithm it predicts the forecasting utilization of resource as graphical representation with bell-shape time algorithm for dynamic abnormal metrics, as shown in below figure

\section{Benefits of Capacity Planner}

vCloud capacity planner delivers the increased productivity of server consolidation and reduced complexity through IT standardization and improved predictability with capacity utilization trends and virtualiztion.

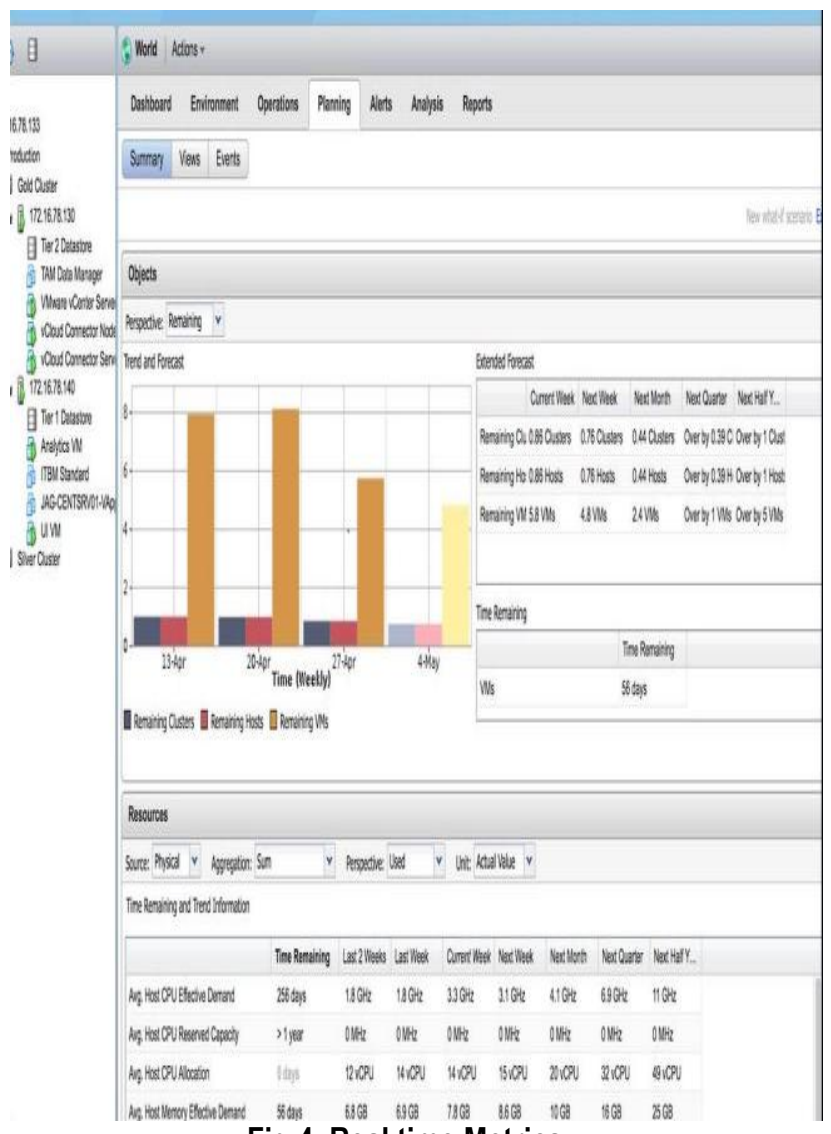

Fig 4. Real time Metrics

\section{CONCLUSION AND FUTURE WORK}

The vCloud Analytics for Capacity Planner product could be leveraged to offer high values assessment services for the data centers. The data centers like Consolidation estimates which are optimized for conducting estimates for the set of activities which are take before the customer acquired. This consolidation estimates give the guidance for what is achieved through virtualization and consolidation assessments. This would gives simplified workflow for VCapacity planner which has a defined methodology to conduct more estimates. The Consultants and professional service organizations are wanted to conduct a deep dive analysis for their customers which is known Capacity assessments. The capacity assessments are provide a detailed route for how its customers can achieve an optimized, virtualized data center. VCloud Capacity assessments shows the implementation blueprint for the state of its data center.

\section{REFERENCES}

[1].Bu-Sung Lee, Dusit Niyato and Sivadon Chaisiri (2012) "Optimization of resource provisioning cost in cloud computing", IEEE Transactions On Services Computing, Vol.5, No.2, pp.164-177. 
[2].A. Ashraf, "Cost-Efficient Virtual Machine Provisioning for Multitier Web Applications and Video Transcoding", Proceedings of the IEEE/ACM International Symposium on Cluster, Cloud and Grid Computing, (2013) May 13-16; Delft, Netherlands.

[3]. D. G. Feitelson, "Parallel workloads archive:Logs",(2014),http://www.cs.huji.ac.il/labs/parallel/workload/l_i ntel_netbatch/index.html

[4]. A. Ashraf, B. Byholm and I. Porres, "CRAMP: Cost-efficient Resource Allocation for Multiple web applications with Proactive scaling", Proceedings of the IEEE International Conference on Cloud Computing Technology and Science, (2012) December 3-6; Taipei, Taiwan.

[5]. Y. Choi and Y. Lim, "A Study on Dynamic VM Provisioning Approach in Cloud Computing", Proceedings of the International Conference Smart Technologies for Energy, Information and Communication, (2014) August 5-6; Narashino, Japan.

[6]. Y. Jiang, C.-S. Perng, T. Li, and R. Chang, "Intelligent cloud capacity management," in 2012 NOMS.

[7]. W. Peng, H. Wang, M. Liu, and W. Wang, "An algorithmic approach toevent summarization," in Proc. 2010 SIGMOD.

[8]. D. Patnaik, M. Marwah, R. Sharma, and N. Ramakrishnan, "Sustainableoperation and management of data center chillers using temporal datamining," in 2009 KDD.

[9]. S. Mylavarapu, V. Sukthankar, and P. Banerjee, "An optimized capacityplanning approach for virtual infrastructure exhibiting stochastic workload,"in 2010 SAC.

[10].Z. Shen, S. Subbiah, X. Gu, and J. Wilkes, "Cloudscale: elastic resourcescaling for multi-tenant cloud systems," in 2011 SoCC.

[11].Y. Jiang, C. Perng, T. Li and R. N. Chang, "Cloud analytics for capacity planning and instant VM provisioning", IEEE Transactions on Network and Service Management, vol. 3, (2013), pp. 312- 325.

[12]. A. Ashraf, B. Byholm and I. Porres, "A Session-Based Adaptive Admission Control Approach for Virtualized Application Servers", Proceedings of the IEEE International Conference on Utility and Cloud Computing, (2012) November 5-8; Chicago, USA.

[13]. J. Almeida, D. Ardagna and C. Francalanci, "Resource Management in the Autonomic Service- Oriented Architecture", Proceedings of the IEEE International Conference on Autonomic Computing, (2006) June 13-16; Dublin, Ireland.

[14]. B. Abrahao, V. Almeida, J. Almeida, A. Zhang, D. Beyer and F. Safai, (Eds.), "Self-adaptive SLA-driven capacity management for internet services", Proceedings of the 10th IEEE/IFIP on Network Operations and Management, (2006) April 03-07; New York, U.S.

[15]. A. Gandhi, M. Harchol-Balter, R. Raghunathan, and M. A. Kozuch,"Autoscale: dynamic, robust capacity management for multitier datacenter," ACM Trans. Computer Systems, 2011.
[16]. Y. Jiang, C.-S. Perng, T. Li, and R. Chang, "Self-adaptive cloud capacityplanning," in 2012 SCC.

[17]. Y. Jiang, C.-S. Perng, T. Li, and R. Chang, "ASAP: a selfadaptiveprediction system for instant cloud resource demand provisioning," in2011 ICDM.

[18]. D. Niyato, A. V. Vasilakos and Z. Kun, "Resource and Revenue Sharing with Coalition Formation of Cloud Providers: Game Theoretic Approach", Proceedings of the IEEE/ACM International Symposium on Cluster, Cloud and Grid Computing, (2011) May 23-26; Newport beach, USA.

[19]. S. Meng, L. Liu, and V. Soundararajan, "Tide: achieving selfscaling invirtualized datacenter managment middleware," in 2010 MiddleWare.

[20]. Y. Jiang, C.-S. Perng, and T. Li, "Natural event summarization," in 2011CIKM.

[21]. G. Tesauro, N.K. Jong, R. Das, and M.N. Bennani,"A Hybrid Reinforcement Learning Approach to AutonomicResource Allocation," Proc. IEEE Intl. Conf.Autonomic Computing (ICAC'06), Dublin, Ireland, June 13-16, 2006.

[22]. D. Thain, T. Tannenbaum, and M. Livny, "DistributedComputing in Practice: The Condor Experience,"Concurrency and Computation: Practice and Experience,Vol. 17, No. 2-4, pages 323-356, FebruaryApril, 2005.

[23]. D.A. Menasc'e and M.N. Bennani, "On the Use ofPerformance Models to Design Self-Managing ComputerSystems," Proc. 2003 Computer MeasurementGroup Conference, Dallas, TX, Dec. 7-12, 2003.

[24]. D.A. Menasc'e and E. Casalicchio, "A Framework forResource Allocation in Grid Computing," Proc. 12thnnual Meeting of the IEEE/ACM InternationalSymposium on Modeling, Analysis, and Simulationof Computer and Telecommunication Systems(MASCOTS), Volendam, The Netherlands, October5-7, 2004.

[25]. Bu-Sung Lee, Dusit Niyato and Sivadon Chaisiri (2012) "Optimization of resource provisioning cost in cloud computing", IEEE Transactions On Services Computing, Vol.5, No.2, pp.164-177.

[26].A.V.K.Shanthi, Y. Sai Pramoda, "Survey on Secure Deduplication and Various Attacks in Cloud", International Journal of Applied Engineering Research ,ISSN 0973-4562 Volume 9, Number 21 (2014) pp. 11307-11314.

[27]. A.V.K.Shanthi, G. Vivek, "A Survey on Fine-Grained Access in Cloud Computing" , International Journal of Applied Engineering Research ISSN 0973-4562 Volume 9, Number 21 (2014) pp. 1043910444. 